\title{
Magnetic Interaction in Ultra-compact Binary Systems
}

\author{
Kinwah $\mathrm{Wu}$ \\ Mullard Space Science Laboratory, University College London, Holmbury St. Mary, Surrey RH5 6NT, \\ United Kingdom
}

\begin{abstract}
This article reviews the current works on ultra-compact double-degenerate binaries in the presence of magnetic interaction, in particular, unipolar induction. The orbital dynamics and evolution of compact white-dwarf pairs are discussed in detail. Models and predictions of electron cyclotron masers from unipolar-inductor compact binaries and unipolar-inductor white-dwarf planetary systems are presented. Einstein-Laub effects in compact binaries are briefly discussed.
\end{abstract}

Key words: stars: binaries: close - stars: white dwarfs - gravitational waves - masers radio continuum: stars $-\mathrm{X}$-rays: binaries - planetary systems

\section{INTRODUCTION}

A binary could be a double-star system, star-planet system or planet-moon system in which two objects revolve around each other under gravity. For binaries with a circular orbit, the separation $a$ of the two components and the orbital period $P_{\mathrm{o}}$ are related by

$$
\begin{aligned}
a & =\left[G\left(M_{1}+M_{2}\right)\left(\frac{P_{\mathrm{o}}}{2 \pi}\right)^{2}\right]^{1 / 3} \\
& \approx 1.1 \times 10^{10}\left(\frac{M_{1}+M_{2}}{\mathrm{M}_{\odot}}\right)^{1 / 3}\left(\frac{P_{\mathrm{o}}}{600 \mathrm{~s}}\right)^{2 / 3} \mathrm{~cm},
\end{aligned}
$$

where $M_{1}$ and $M_{2}$ are the masses of the primary and secondary components respectively, and $G$ is the gravitational constant. (Hereafter the subscripts "o", "1" and "2" represent the orbit, the primary star and the secondary star respectively.) If the secondary component fills its Roche lobe, its mean density $\bar{\rho}_{2}$ is determined by the orbital period:

$$
\bar{\rho}_{2} \approx 3.9 \times 10^{3} \lambda(q)\left(\frac{P_{\mathrm{o}}}{600 \mathrm{~s}}\right)^{-2} \mathrm{~g} \mathrm{~cm}^{-3},
$$

where $\lambda(q)$ is a numerical factor of the order of unity, weakly dependent on the mass ratio $q=M_{2} / M_{1}$ (Eggleton 1983; see also Pacyznski 1971). In a stellar binary with $P_{\mathrm{o}} \approx 600 \mathrm{~s}$, the density of the secondary star would exceed that of a main-sequence star with the same mass. The primary star, which is more massive, is even denser. Thus, stellar binaries with $P_{\mathrm{o}}<600$ s must contain either degenerate stars or black holes, and these short-period systems are known as ultra-compact double-degenerate systems (UCDs).

In principle, UCDs may contain any combinations of white dwarfs, neutron stars or black holes. However, the formation of double white dwarfs are more favourable in the evolutionary channels (see Han 1998; Nelemans et al. 2001; Belczynski \& Taam 2004; Postnov \& Yungelson 2006; Belczynski et al. 2008), and compact double white dwarfs are expected to be more abundant than compact binaries with other combinations of white dwarfs, neutron stars and black holes. Observationally, many compact whitedwarf pairs have been discovered (see Roelof, Nelemans \& Groot 2007), and these system are populous 
in the Milky Way. In this article the main focus will be on double white-dwarf systems. Hereafter, unless otherwise stated, the term UCD will be used solely for short-period systems with two white dwarfs.

Almost all celestial bodies possess a certain magnetism. A substantial fraction of white dwarfs are known to have a magnetic field with strength exceeding $10^{6} \mathrm{G}$ (Chanmugam 1992; Schmidt \& Smith 1995; Wickramasinghe \& Ferrario 2000). The magnetic moments of these white dwarfs are above $10^{32} \mathrm{G} \mathrm{cm}^{3}$. For an orbital separation $<10^{10} \mathrm{~cm}$, these white dwarfs will exert a magnetic field of the order of $\mathrm{kG}$ at the surface of their companion stars. As the two white dwarfs in UCDs are in very close proximity, electromagnetic interaction is inevitable. This alters the orbital dynamics of the binary and gives rise to a variety of unusual observational consequences.

Magnetic interaction between two gravitationally bound celestial objects is common on all scales. A well known example in our backyard is Jupiter and its moon Io. It is believed that Io has a highly conductive core. When Io revolves around Jupiter, it traverses the Jovian magnetic field and a large e.m.f. is created via a unipolar-induction process (Piddington \& Drake 1968; Goldreich \& Lynden-Bell 1969). This e.m.f. drives the flow of electric currents between Jupiter and Io. Observations have shown a hot spot at the polar surface of Jupiter (Clarke et al. 1996), which is identified as the location of foot-points of the magnetic field lines leading to Io. Dissipation of the electric currents in the Jovian atmosphere lights up the foot-points of the magnetic field lines that connect the two objects. On stellar scales, strong magnetic interactions are found between the two stars in RS CVn binaries and in AM Herculis binaries. There is also evidence that substantial magnetic interaction occurs in Algol binaries as well (Richards \& Albright 1993; Retter, Richards \& Wu 2005). In RS CVn binaries the magnetic interaction leads to enhanced coronal activity in the component stars (Uchida \& Sakurai 1983; Ferreira \& Mendoza-Briceño 2005). In AM Herculis binaries, magnetic interaction essentially defines the characteristics of the system. It locks the entire system to into synchronous rotation (Campbell 1983, 1999; Wickramasinghe \& Wu 1991; Wu \& Wickramasinghe 1993); it governs their orbital evolution ( $\mathrm{Li}$, Wu \& Wickramasinghe 1994a, b; Davis et al. 2008); and it determines the hydrodynamics of mass flow from the Roche-lobe spilling low-mass donor star to the magnetic white dwarf primary (Chanmugam \& Wagner 1977; Visvanathan \& Wickramasinghe 1981, see also Warner 1995; Wu 2000).

It is natural that the white dwarfs in UCDs interact magnetically, provided that one or two of the white dwarfs have a sufficiently large magnetic moment. In this article we will review the current research progress on magnetically interacting UCDs and associated systems. We organise the article as follows. In $\S 2$ we discuss the general orbital dynamics of UCDs in compact binaries. In $\S 3$ we present the basics of the unipolar induction model for compact white-dwarf pairs, and in $\S 4$ we discuss the orbital evolution of compact binaries in the presence of unipolar induction. In $\S 5$ we show that unipolar-inductor white-dwarf pairs could be electron-cyclotron maser sources. In $\S 6$ we show how some physics in UCDs can be applied to related systems, such as white dwarf-planet systems, and that magnetically interacting ultra-compact binaries may exhibit Einstein-Laub effects.

\section{ORBITAL DYNAMICS IN COMPACT BINARIES}

AM CVn binaries are the better studied UCD (Solheim 1995; Nelesmans 2005). Mass transfer occurs in AM CVn binaries when the less massive white dwarf overfills its Roche lobe. The in-falling material forms an accretion disk around the white-dwarf primary. If the binary orbit is too compact, the formation of an accretion disk might be prohibited. Mass transfers directly via a gas stream from the inner Lagrangian point of the secondary white dwarf to the surface of the primary white dwarf (Marsh \& Steeghs 2002, see also Wood 2009). The mass transfer dynamics of these double white dwarfs are analogous to those of the Algol binaries. These binaries are known as direct-impact mass-transfer double degenerates. The orbital dynamics and evolution of AM CVn binaries and direct-impact mass-transfer double degenerates are regulated by the mass transfer process. Their high-energy emissions, such as X-rays, are accretion powered. Magnetic interacting UCDs are similar to AM CVn binaries and direct-impact mass-transfer double degenerates, as they also have two white dwarfs revolving around each other in a very tight orbit. However, they are different from those binaries in that magnetic interaction governs the angular momentum redistribution, and that internal energy dissipation within the system plays an important role in regulating the orbital dynamics and hence the orbital evolution. 


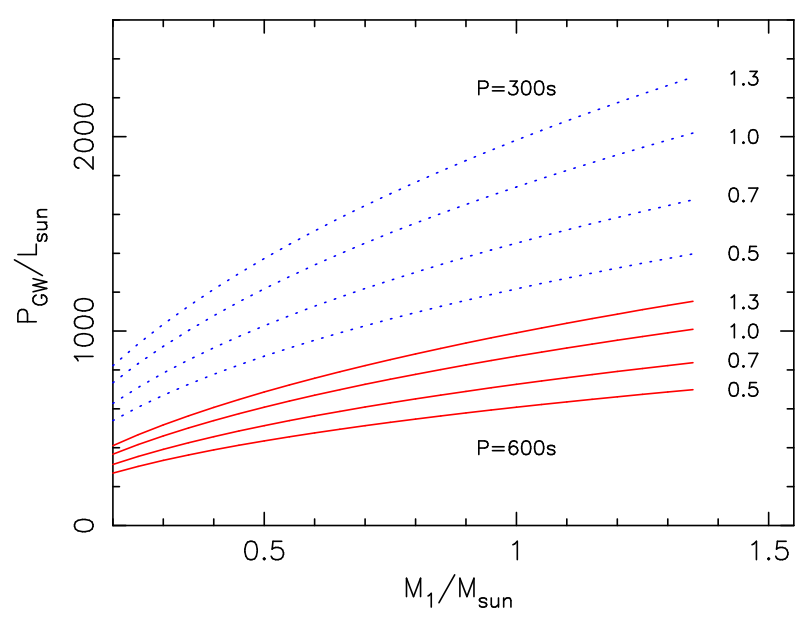

Fig. 1 The power of gravitational waves from white-dwarf pairs, normalised to the solar bolometric luminosity, as a function of the primary white-dwarf mass $M_{1}$, for orbital periods of $600 \mathrm{~s}$ (solid lines) and $300 \mathrm{~s}$ (dotted lines). Each curve corresponds to a value of the secondary whitedwarf mass, labeled in solar-mass unit. (Adapted from Wu, Ramsay \& Willes (2008).)

UCDs are strong sources of gravitational waves because of their compact orbits. The power of their gravitational radiation (assuming an orbital eccentricity $e=0$ ) is

$$
\begin{aligned}
\dot{E}_{\mathrm{gw}} & =-\frac{32}{5} \frac{G^{4}}{c^{5}} \frac{M_{1}^{2} M_{2}^{2}\left(M_{1}+M_{2}\right)}{a^{5}} \\
& =-\frac{32}{5} \frac{G^{7 / 3}}{c^{5}} M_{\mathrm{chirp}}^{10 / 3} \omega_{\mathrm{o}}^{10 / 3} \\
& =-1.2 \times 10^{36}\left[\left(\frac{M_{\mathrm{chirp}}}{\mathrm{M}_{\odot}}\right)\left(\frac{600 \mathrm{~s}}{P_{\mathrm{o}}}\right)\right]^{10 / 3} \mathrm{erg} \mathrm{s}^{-1}
\end{aligned}
$$

(see Landau \& Lifshitz 2002), where $c$ is the speed of light. The chirp mass $M_{\text {chirp }}=\tilde{M}^{3 / 5}\left(M_{1}+M_{2}\right)^{2 / 5}$, where $\tilde{M} \equiv M_{1} M_{2} /\left(M_{1}+M_{2}\right)$ is the reduced mass of the binary. It relates the orbital angular momentum $J_{\mathrm{O}}$ to the orbital angular velocity $\omega_{\mathrm{O}}$ via

$$
J_{\mathrm{o}}=G^{2 / 3} M_{\text {chirp }}^{5 / 3} \omega_{\mathrm{o}}^{-1 / 3} .
$$

For UCDs with $P_{\mathrm{o}} \sim 600 \mathrm{~s}$ or shorter, the power of the gravitational radiation greatly exceeds the solar power in the electromagnetic spectrum (Fig. 11).

The orbital angular momentum of a binary system is given by $J_{\mathrm{o}}=M_{1} M_{2} a^{2} \omega_{\mathrm{o}} /\left(M_{1}+M_{2}\right)$. The orbital separation $a$ and the orbital angular velocity $\omega_{\mathrm{o}}$ are related by $\omega_{\mathrm{o}}^{2}=\left(2 \pi / P_{\mathrm{o}}\right)^{2}=G\left(M_{1}+M_{2}\right) a^{-3}$. The evolution of the binary orbit is determined by redistribution of angular momentum within the system and the loss of angular momentum from the system. These processes are described by the following coupled differential equations:

$$
\begin{aligned}
& \frac{\dot{\omega}_{\mathrm{o}}}{\omega_{\mathrm{o}}}=\left(3-\frac{M_{1}}{M_{1}+M_{2}}\right) \frac{\dot{M}_{1}}{M_{1}}+\left(3-\frac{M_{2}}{M_{1}+M_{2}}\right) \frac{\dot{M}_{2}}{M_{2}}-3 \frac{\dot{J}_{\mathrm{o}}}{J_{\mathrm{o}}}, \\
& \frac{\dot{\omega}_{1}}{\omega_{1}}=-\left(1+\frac{2}{n_{1}}\right) \frac{\dot{M}_{1}}{M_{1}}+\frac{\dot{J}_{1}}{J_{1}},
\end{aligned}
$$




$$
\frac{\dot{\omega}_{2}}{\omega_{2}}=-\left(1+\frac{2}{n_{2}}\right) \frac{\dot{M}_{2}}{M_{2}}+\frac{\dot{J}_{2}}{J_{2}}
$$

where " ." denotes time derivatives. The derivation of the above equations has assumed that $M_{1} \propto R_{1}^{n_{1}}$ and $M_{2} \propto R_{2}^{n_{2}}$, where $n_{1,2}$ are the proportional indices in the mass-radius relations of the two stars.

Conservation of angular-momentum requires $\dot{J}=\dot{J}_{\mathrm{o}}+\dot{J}_{1}+\dot{J}_{2}$. When there is no mass loss from the system $\left(\dot{M}=\dot{M}_{1}+\dot{M}_{2}=0\right)$, orbital angular momentum is extracted from the binary only through the emission of gravitational waves. This gives a rate of orbital angular-momentum loss

$$
\begin{aligned}
\dot{J} & =\dot{J}_{\mathrm{gw}} \\
& =-\frac{32}{5} \frac{G^{7 / 2}}{c^{5}} \frac{M_{1}^{2} M_{2}^{2}\left(M_{1}+M_{2}\right)^{1 / 2}}{a^{7 / 2}} \\
& =-\frac{32}{5} \frac{G^{7 / 3}}{c^{5}} M_{\mathrm{chirp}}^{10 / 3} \omega_{\mathrm{o}}^{7 / 3}
\end{aligned}
$$

(Landau \& Lifshitz 2002). If there is no mass change between the two stars $\left(\dot{M}_{1}=\dot{M}_{2}=\dot{M}_{\text {chirp }}=0\right.$ ) and if the stellar spins are decoupled from the orbital rotation, the evolution of the orbital angular frequency is dictated by gravitational radiation loss:

$$
\begin{aligned}
\frac{\dot{\omega}_{\mathrm{o}}}{\omega_{\mathrm{o}}} & =-3 \frac{\dot{J}_{\mathrm{gw}}}{J_{\mathrm{o}}} \\
& =\frac{96}{5} \frac{G^{5 / 3}}{c^{5}} M_{\mathrm{chirp}}^{5 / 3} \omega_{\mathrm{o}}^{8 / 3} .
\end{aligned}
$$

It is clear that in the absence of mass loss from the system and in the absence of angular momentum exchange or mass exchange between the two stars, the binary orbit is always spun up, i.e. the orbital period decreases with time.

If the stellar spins are coupled with the orbital rotation, then angular momenta can be injected from the orbit into the stars. In the 'ideal' case where the two stars and the orbit are locked in synchronous rotation,

$$
\begin{aligned}
\frac{\dot{\omega}_{\mathrm{o}}}{\omega_{\mathrm{o}}} & =-3 \frac{\dot{J}_{\mathrm{gw}}}{J_{\mathrm{o}}}\left[1-\frac{3}{J_{\mathrm{o}}}\left(J_{1}+J_{2}\right)\right]^{-1} \\
& =\frac{96}{5} \frac{G^{5 / 3}}{c^{5}} M_{\mathrm{chirp}}^{5 / 3} \omega_{\mathrm{o}}^{8 / 3}\left[1-\frac{3\left(M_{1} R_{1}^{2}+M_{2} R_{2}^{2}\right) \omega_{\mathrm{o}}^{4 / 3}}{G^{2 / 3} M_{\mathrm{chirp}}^{5 / 3}}\right]^{-1} .
\end{aligned}
$$

Internal energy dissipation in the system is unimportant in an ideal, perfectly synchronous rotating system. When $M_{\text {chirp }}$ is fixed, $\omega_{\mathrm{o}} \propto J_{\mathrm{o}}^{3}$. As additional angular momentum is extracted from the orbit to spin up the two stars, the orbital angular frequency will accelerate further when the system loses energy via gravitational radiation. This gives larger values for $\dot{\omega}_{\mathrm{o}}$ than those in the case where the spins of the star and the orbital rotation are decoupled (cf. Eq. 9 and 10).

In reality, perfect synchronism is hard to achieve for any binary system. Although AM Herculis binaries are supposed to be magnetically locked into synchronous rotation, there are a small fraction (e.g. the system BY Cam, Mason et al. 1998) in which the white dwarf rotates asynchronously with the orbital motion. The situation is similar for UCDs. There should be certain spin-orbit asynchronism despite the fact that strong tidal force and magnetic interaction tend to synchronise the star spins and orbital rotation. When there are internal energy dissipation and angular momentum redistribution in the system, the formulation for the orbital evolutionary dynamics described above would need modifying. In the next section we will discuss the case of slightly asynchronous UCDs in which magnetic interaction mediates the angular momentum exchange between the stars and the orbit. Also, there is no mass transfer between the stars in these systems, contrary to the magnetically locked AM Herculis binaries. The dynamics would be more complicated when mass exchange occurs, and when the system loses mass. (Orbital evolution of binaries under mass exchange and mass outflow were discussed, for example, in Wu 1997.) 


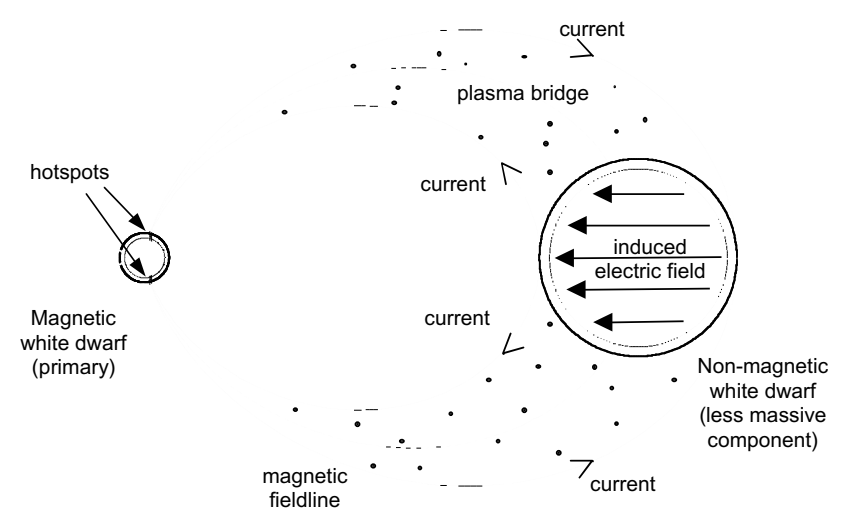

Fig. 2 A schematic illustration of the unipolar-inductor model for white-dwarf pairs. As the system revolves, a large e.m.f. is induced across the non-magnetic white dwarf and currents are driven between the two stars. The resistance in the atmospheric layers of the white dwarfs causes energy dissipation. Electromagnetic waves are emitted from the heated white-dwarf atmosphere.

\section{UNIPOLAR INDUCTION IN COMPACT BINARIES}

The small separation between the stars in a UCD allows electromagnetic interactions to occur between them. One possible process is unipolar induction, which could generate strong electric currents between the two white dwarfs, as well as large Lorentz torques on the orbit and the stars.

Unipolar induction is a fundamental electrodynamic process. It is a manifestation of Maxwell's equations and the Lorentz force acting on electrons (Feynman, Leighton \& Sands 1964; Assis 2000). Its validity is verified by laboratory experiments (see Miller 1981; Kelley 1999). A proper interpretation of unipolar induction is still under discussion, as there are subtleties in how it is related to electrodynamics and relativity (see, for example, recent articles by Montgomery 1999; Guala-Valverde, Mazzoni \& Achilles 2002). A well known example of astrophysical unipolar inductors is the Jupiter-Io system (Piddington \& Drake 1968; Goldreich \& Lynden-Bell 1969). It has been proposed that unipolar induction operates in pulsar magnetospheres (Goldreich \& Julian 1969), in magnetic binary stars (e.g. AM Herculis binaries, Chanmugam \& Dulk 1982), in stellar-planetary systems (see e.g. Zarka 2007; Laine, Lin \& Dong 2008), in white-dwarf planetary systems (Li, Ferrario \& Wickramasinghe 1998; Willes \& Wu 2004), and in magnetised accretion disks around black holes (Shatskii 2003, see also Punsly 2001; Komissarov 2004). There are also models wherein cosmic ray particles are accelerated to ultra-high energies via unipolar induction (Chanmugam \& Brecher 1985; Shatskii \& Karashev 2002; see also discussions in Blandford 2000). A unipolar-inductor model (sometimes known as electric-star model) for UCD was proposed (Wu et al. 2002; Ramsay et al. 2002; Willes, Wu \& Kuncic 2004; Dall'Osso, Israel \& Stella 2006, 2007; Wu, Ramsay \& Willes 2008) to explain the peculiar properties of the X-ray sources RX J1914+24 and RX J0806+15.

\subsection{Compact white-dwarf pairs}

When a non-magnetic conducting body of linear size $R$ traverses a magnetic field $\boldsymbol{B}$ with a velocity $\boldsymbol{v}$, an e.m.f. $\Phi \sim R|\boldsymbol{E}|$ is induced across the conducting body, where $\boldsymbol{E}=\boldsymbol{\beta} \times \boldsymbol{B}$ and $\boldsymbol{\beta}=\boldsymbol{v} / c$. This is the basic principle of the operation of unipolar-induction in magnetically interacting white-dwarf pairs. The setting for a unipolar inductor UCD is illustrated in Figure 2] The e.m.f. across the non-magnetic white dwarf in 
orbit with a magnetic white dwarf is therefore

$$
\begin{aligned}
\Phi & \approx \frac{2 \pi}{c}\left(\frac{\mu_{1} R_{2}}{a^{2} P_{\mathrm{o}}}\right)(1-\alpha) \\
& =\left(\frac{\mu_{1} R_{2}}{c}\right)\left(\frac{2 \pi}{P_{\mathrm{o}}}\right)^{7 / 3}(1-\alpha)\left[G M_{1}(1+q)\right]^{-2 / 3},
\end{aligned}
$$

where $q$ ( $\equiv M_{2} / M_{1}$ ) is the mass ratio of the non-magnetic to the magnetic white dwarf, $R_{2}$ and $R_{1}$ are the respective radii of the two white dwarfs, and $\mu_{1}$ is the magnetic moment of the magnetic white dwarf. The induced e.m.f. depends on the degree of synchronism between the spin of the magnetic white dwarf and the orbit. Without loss of generality we may specify the degree of asynchronism using a parameter $\alpha$, which is the ratio of the spin angular speed of the magnetic white dwarf $\omega_{1}$ to the orbital angular speed $\omega_{\mathrm{o}}$. (We consider the convention in which the anti-clockwise direction is positive.)

Provided that the space between the white dwarfs is permeated by some plasma, the e.m.f. will drive electric currents, which flow along the magnetic field lines connecting the two white dwarfs. Although white dwarfs have a highly conducting core, there is substantial electric resistance in the white-dwarf atmosphere, where electrical dissipation occurs. The total power generated by the current dissipation in the two stars is

$$
\begin{aligned}
W & =I^{2}\left(\mathcal{R}_{1}+\mathcal{R}_{2}\right) \\
& =\frac{\Phi^{2}}{\mathcal{R}_{1}+\mathcal{R}_{2}},
\end{aligned}
$$

where $I$ is the total current, and $\mathcal{R}_{1}$ and $\mathcal{R}_{2}$ are the effective resistance of the magnetic and the non-magnetic white dwarf respectively. For an object with a length $L$ and a cross-sectional area $A$, the resistance is simply $\mathcal{R}=L / A \sigma$ (with $\sigma$ as electric conductivity). It follows that the ratio of the effective resistances of the white dwarfs is

$$
\frac{\mathcal{R}_{1}}{\mathcal{R}_{2}} \sim\left(\frac{\sigma_{2}}{\sigma_{1}}\right)\left(\frac{R_{2}^{2}}{f R_{1}^{2}}\right)\left(\frac{\Delta h_{1}}{\Delta h_{2}}\right),
$$

where $\sigma_{1}$ and $\sigma_{2}$ are the corresponding electric conductivities of the two white dwarfs, $\Delta h_{1}$ and $\Delta h_{2}$ are the thicknesses of the dissipative surface layers of the white dwarfs, and $f$ is the fractional effective area of the magnetic poles (hot spots) on the surface of the magnetic white dwarf. As $f \ll 1$ (see Wu et al. 2002), the effective resistance of the magnetic white dwarf is significantly larger than that of the non-magnetic white dwarf.

As the electric currents pass through both white dwarfs, the ratio of the power dissipation in the magnetic primary to that of the non-magnetic secondary is $W_{1} / W_{2}=\mathcal{R}_{1} / \mathcal{R}_{2}$. Taking account of the geometry of the current loops, we obtain

$$
\begin{aligned}
\frac{W_{1}}{W_{2}} & \approx \zeta\left(\frac{\sigma_{2}}{\sigma_{1}}\right)\left(\frac{R_{2}}{\Delta R_{2}}\right)\left[\frac{G\left(M_{1}+M_{2}\right)}{R_{1}^{3}}\left(\frac{P_{\mathrm{o}}}{2 \pi}\right)^{2}\right]^{1 / 2} \\
\mathcal{R}_{1} & \approx \frac{1}{2 \sigma_{1}}\left(\frac{H}{\Delta d}\right)\left(\frac{a}{R_{1}}\right)^{3 / 2} \frac{\mathcal{J}(e)}{R_{2}} \\
\mathcal{R}_{2} & \approx \frac{4}{\pi \sigma_{2}}\left(\frac{\Delta R_{2}}{R_{2}^{2}}\right)
\end{aligned}
$$

(see Appendices $\mathrm{A}$ and $\mathrm{B}$ of $\mathrm{Wu}$ et al. (2002) for details), where $\Delta R_{2}$ is the thickness of the secondary's atmosphere and $\zeta$ is a structure factor of the order of unity. The factor $\mathcal{J}(e)$ depends on the radii of the white dwarfs relative to the orbital separation. Its value is of the order of unity for white-dwarf pairs with $P_{\mathrm{o}}$ less than an hour.

The electric conductivity of plasma at an electron temperature $T_{\mathrm{e}}$ is given by

$$
\sigma=\gamma\left(\frac{2^{5 / 2}}{\pi^{3 / 2}}\right) \frac{\left(k T_{\mathrm{e}}\right)^{3 / 2}}{m_{\mathrm{e}}^{1 / 2} Z e^{2} \ln \Lambda},
$$



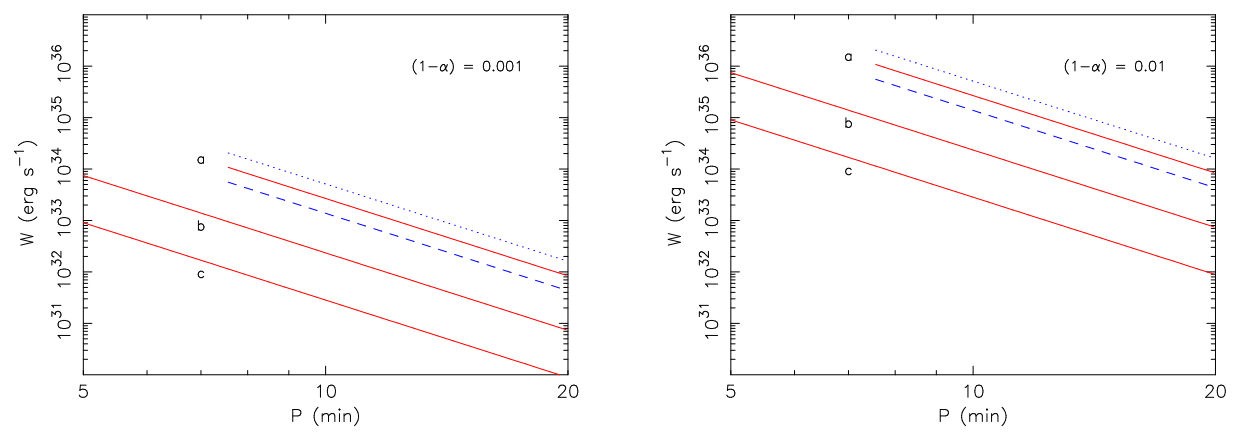

Fig. 3 The total power generated by the dissipation of electric currents as a function of the orbital period for spin-orbit asynchronism $(1-\alpha)$ of $1 / 1000$ and $1 / 100$ (left and right panels respectively) predicted by the unipolar induction for UCDs. The solid lines correspond to cases with a 1.0-M $\odot$ magnetic white dwarf. Lines $\mathrm{a}, \mathrm{b}$ and $\mathrm{c}$ correspond to the cases with a non-magnetic companion white dwarf of $0.1,0.5$ and $1.0 \mathrm{M}_{\odot}$ respectively. The dotted line corresponds to the case with a $0.7-\mathrm{M}_{\odot}$ magnetic white dwarf and a $0.1-\mathrm{M}_{\odot}$ non-magnetic white dwarf; the dashed line, a 1.3$\mathrm{M}_{\odot}$ magnetic white dwarf and a 0.1-M $\mathrm{M}_{\odot}$ non-magnetic white dwarf. The white-dwarf magnetic moments are $10^{32} \mathrm{G} \mathrm{cm}^{3}$ in all cases. (Adapted from Wu, Ramsay \& Willes (2008).)

(Spitzer \& Härm 1953) where $k$ is the Boltzmann constant, $m_{\mathrm{e}}$ is the electron mass, $e$ is the electron charge, $Z$ is the ion charge number, and $\ln \Lambda$ is the Coulomb logarithm. The factor $\gamma$ depends on $Z$, which has values between $0.6(Z=1)$ and $1(Z \rightarrow \infty)$ (see Alfvén \& Fälthammar 1963). For a white-dwarf atmosphere with $T_{\mathrm{e}} \sim 10^{5} \mathrm{~K}$, the conductivity $\sigma \sim 10^{13}-10^{14}$ esu. Since the conductivities of the atmospheres of the white dwarfs are similar to each other, the majority of the electrical power will be dissipated in small regions at the footpoints of the current-carrying field lines on the surface of the magnetic white dwarf.

The operation of a unipolar inductor in UCDs can be understood in terms of an electric circuit model. The non-magnetic white dwarf, where the e.m.f. is generated, acts as an electric generator or a battery (with a small internal resistance); the plasmas that mediate the currents are the conducting circuit wires; and the magnetic white dwarf is the resistive load, where most of the dissipation occurs. The induced e.m.f. depends strongly on the binary orbital period, the degree of spin-orbit synchronism, and the mass (radius) of the non-magnetic white dwarf. The resistivities within the circuit, however, depend also on the internal properties of the white-dwarf atmosphere. For a large range of mass ratios, unipolar induction in a compact white-dwarf pair can produce luminosities similar to or larger than the Sun, requiring only a small degree of spin-orbit asynchronism (Fig. 3).

The remaining question now is: what actually drives the electric currents? The energy reservoir is in fact the binary orbit. Through unipolar induction, a back Lorentz torque is generated and it acts on the orbit. Orbital energy is extracted, which provides the e.m.f. for the current circuit. Thus, similar to accretion, the ultimate energy source in a unipolar-inductor white-dwarf pair is still the gravitational potential.

\subsection{Candidate unipolar-inductor ultra-compact double degenerates}

The two candidate unipolar-inductor UCDs, RX J1914+24 and RX J0806+15, are short-period variable X-ray sources discovered in the ROSAT observations (Motch et al. 1996; Cropper et al. 1998; Israel et al. 1999). One of their remarkable characteristics is that only a single period is shown in the variations across the electromagnetic spectrum - from the infra-red (IR) and optical to X-ray bands (see Fig. 4]and 5). The period of RX J1914+24 is $569 \mathrm{~s}$ (Ramsay et al. 2002), and the period of RX J0806+15 is $321 \mathrm{~s}$ (Israel et al. 2003). Their X-ray light curves show pulse-like profiles, suggesting that the emission originates from a hot spot on the surface of one of the stars. The optical/IR light curves, in contrast, show sinusoidal variations, 


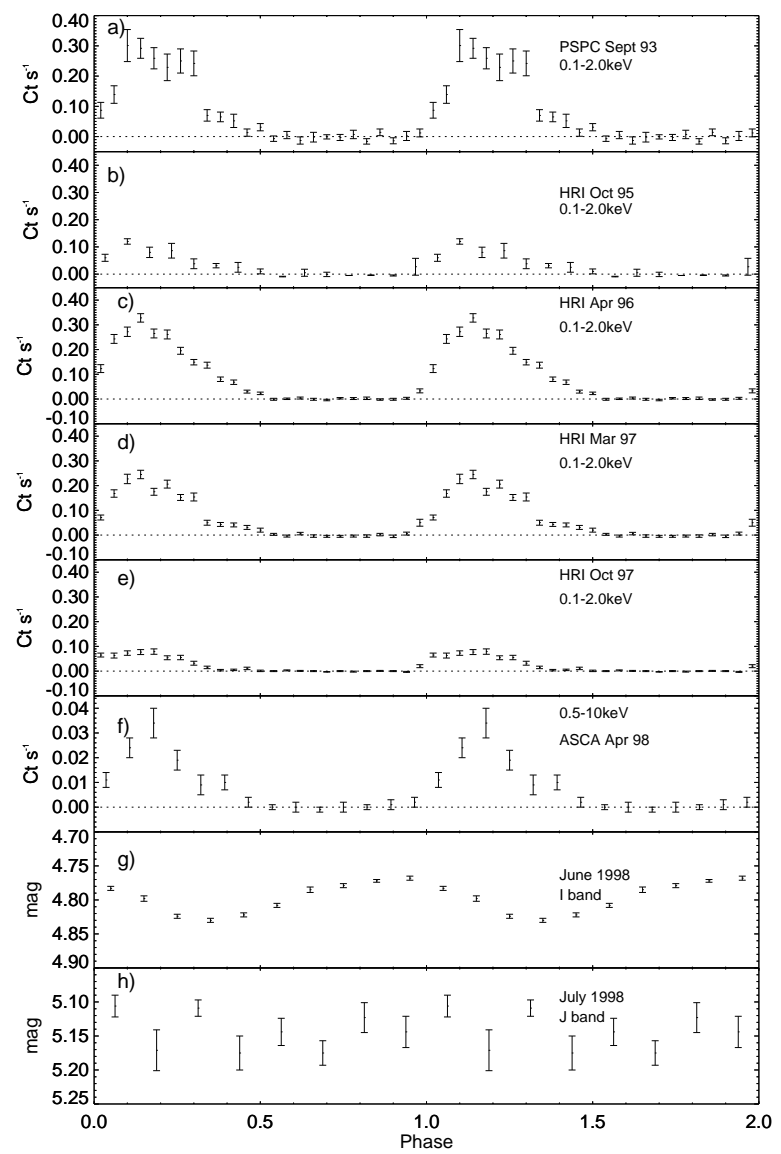

Fig. 4 The folded light curves of RX J1914+24 (Ramsay et al. 2000). Panels (a) to (e) are X-ray light curves obtained by ROSAT; panel (f) is the X-ray light curve obtained by ASCA. Panels (g) and $(\mathrm{h})$ are the I and $\mathbf{J}$ band near-IR light curves obtained by UKIRT.

and variations are anti-phased with the variations in the X-ray bands. The optical/IR emission region is therefore extensive and not coincident with the X-ray emitting region.

The nature of RX J1914+24 and RX J0806+15 has been under debate. It is now generally accepted that they are binary systems with orbital periods of $569 \mathrm{~s}$ and $321 \mathrm{~s}$ respectively. This requires the two component stars in RX J1914+24 and RX J0806+15 to be degenerate stars. Moreover, they are very compact binaries with orbital separations similar to Jupiter's linear size. Several models for them been proposed: (i) face-on intermediate polar (IP) (Norton, Haswell \& Wynn 2004), (ii) degenerate polar (degenerate AM Herculis binary) (Cropper et al. 1998), (iii) direct impact accretor (Marsh \& Steeghs 2002; Ramsay et al. 2002), (iv) neutron star-white dwarf pair (Ramsay et al. 2002), and (v) unipolar-inductor binary (Wu et al. 2002; Dall'Osso, Israel \& Stella 2006, 2007). In the first four models accretion is the energy source for the observed X-rays. The unipolar-inductor model, however, suggested that the emission of X-rays is caused by ohmic dissipation of electric currents in the white-dwarf atmosphere. An assessment of the models can be found in Cropper et al. (2004). 


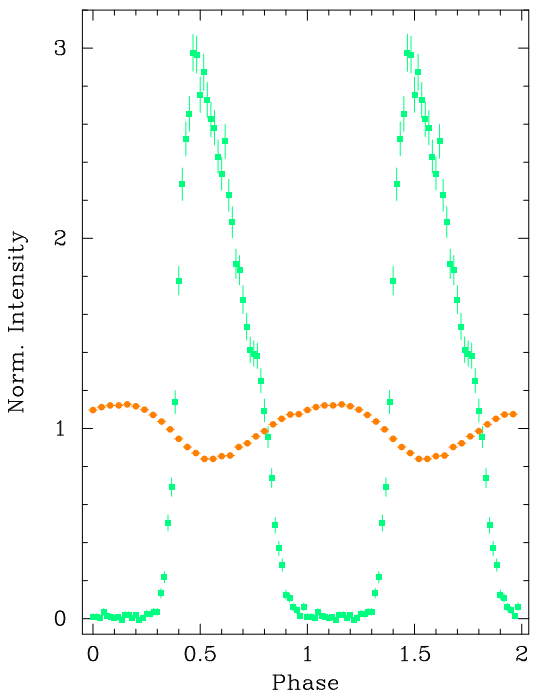

Fig. 5 The folded Chandra X-ray and VLT optical R-band light curve of RX J0806+15 (provided by G. L. Israel).

In the face-on IP model, a moderately magnetized white dwarf is accreting material from a mainsequence donor star. The white-dwarf spin is not synchronous with the orbital rotation. The pulse period of the X-ray emission is the white-dwarf spin period, which is much shorter than the undetected orbital period. In the degenerate polar model, the accreting white dwarf has a strong magnetic field. The mass-donor white dwarf may or may not be magnetic. The whole system is locked into synchronous rotation by a white-dwarf magnetic field as in the usual polars (AM Herculis binaries). The observed period is the spin periods of the two white dwarfs. It is also the period of the orbital rotation. In the direct impact accretor model, both stars are white dwarfs. Their magnetic fields are irrelevant as they do not play a significant role in determining the emission and the orbital dynamics. The X-ray hot spot is the stream impact point. Its location on the equator of the accreting white dwarf is fixed in the rotational frame of the binary. The observed period is the orbital period and the spin period of the mass-donor white dwarf, but it is not necessarily the spin period of the accreting white dwarf. In the neutron star-white dwarf pair model, there is no mass transfer from the white dwarf to the neutron star. Otherwise, much higher X-ray luminosities would have been observed. There is only a low level of accretion, which is likely sustained by remnant material in the vicinity of the binary ejected in previous evolutionary phases.

In the unipolar-inductor model, RX J1914+24 and RX J0806+15 contain one magnetic and one nonmagnetic (or weakly magnetic) star. It allows the magnetic star to be a neutron star or a white dwarf, but in a restrictive version both stars are white dwarfs. Electromagnetic radiation from these two systems is not powered by accretion. Instead it is due to the dissipation of electric currents. The unipolar-inductor binary is in contrast to other stellar objects whose energy sources are either accretion or nuclear reaction. A small asynchronism between the spin of the magnetic white dwarf and the orbital rotation is required in order to generate a substantial e.m.f. which drives the electric currents. The focusing field lines channel the electric currents toward a small foot-point region on the surface of the primary white dwarf. This gives a very small $\mathrm{X}$-ray emission spot. The optical/IR emission is from a heated hemisphere of the secondary white irradiated by the X-rays emitted from the primary white dwarf. The optical/IR emitting area is therefore extensive. This geometrical configuration naturally leads to an anti-phasing between the optical/IR emission and Xrays. 
In order to account for the observed X-ray luminosity, all accreting white-dwarf models (the face-on IP, degenerate polar and direct-impact accretor model) require a relatively high mass transfer rate. If we take the X-ray luminosity of $\sim 10^{35}-10^{36} \mathrm{erg} \mathrm{s}^{-1}$ (assuming a distance of $100 \mathrm{pc}$ ) deduced for RX J1914+24 from the ROSAT data (Cropper et al. 1998), the mass transfer rate of the system exceeds $5 \times 10^{17} \mathrm{~g} \mathrm{~s}^{-1}$. Transfer of material from the low-mass secondary star to the high-mass primary star, in general, causes the binary orbit to expand, and hence the orbital period increases. For rapid mass transfer on timescales shorter than the timescale of orbital evolution driven by angular momentum loss (via gravitational radiation or magnetic braking), the orbital period of the binary is expected to increase, i.e. $\dot{P}_{\mathrm{o}}>0$ (or $\dot{\omega}_{\mathrm{o}}<0$ ). X-ray timing observations, however, show that the periods of these systems are decreasing (Strohmayer 2002, 2003, 2004, 2005; Hakala et al. 2003; Ramsay et al. 2005), which is inconsistent with the mass-transfer scenario. Accretion models are difficult to reconcile with the findings that RX J1914+24 has an almost featureless optical spectrum (Steeghs et al. 2006) and that RX J0806+15 has only a few very weak optical emission lines (Israel et al. 2002). It is puzzling that signatures of accreting systems such as the strong prominent $\mathrm{H}$ Balmer and He II emission lines as those observed in cataclysmic variables (see Williams 1983) and lowmass X-ray binaries (see Lewin, van Paradijs \& van den Heuvel 1997) are not seen in the optical spectra of RX J1914+24 and RX J0806+15. Recent Chandra observations of RX J0806+15 confirmed that emission lines are absent in the X-ray band (Strohmayer 2008). Another difficulty of the scenarios with a strongly magnetic white dwarf (as in the degenerate polar model) is non-detection of cyclotron harmonic features in the optical spectra (cf. the observed cyclotron humps in the optical spectra of AM Herculis binaries, see e.g. Cropper et al. 1989).

The unipolar-inductor model avoids the above difficulties of the accretion models. Although the model is generally consistent with existing observations (see Cropper et al. 2004), there are some concerns regarding whether or not it is applicable to RX J1914+24 and RX J0806+15, which are presumably compact white-dwarf pairs (e.g. Barros et al. 2005, 2007; Laine, Lin \& Dong 2008; Wood 2009). Some concerns, e.g. regarding the exact magnetic-field geometry and the relative lead/lag in the X-ray pulses and the optical maxima, can be resolved easily. There are, however, several more serious issues. For instance, certain implicit assumptions have been made in order to facilitate the unipolar-induction process. In plasma, the time-dependence of a magnetic field is governed by

$$
\frac{1}{c} \frac{\partial \boldsymbol{B}}{\partial t}=\nabla \times(\boldsymbol{\beta} \times \boldsymbol{B})+\frac{c}{4 \pi \sigma} \nabla^{2} \boldsymbol{B} .
$$

The first term on the right-hand side of the equation is the induction term, and the second term is the diffusion term. In the unipolar-induction model, the diffusion term is omitted based on the assumption that the white dwarf's core is a perfect conductor, i.e., setting the conductivity $\sigma \rightarrow \infty$. This issue was discussed in detail recently by Laine, Lin \& Dong (2008) in the context of binaries containing a normal star and a planet. The assumption is probably acceptable for compact white-dwarf pairs, as the white-dwarfs' cores are practically a fermi ball of electrons. Another serious issue concerns the life-span of unipolar-inductor UCDs. If the system achieves spin-orbit synchronisation on a very short timescale, the unipolar-induction process will be quenched. As the X-rays from unipolar-inductor UCDs are powered by electrical dissipation, a rapid spin-orbit syncronisation would imply that the X-ray active phases of the system are brief. If unipolar induction occurs only in transient episodes, it would play a less important role in determining the orbital evolution of UCDs. In $\S 4.2$ we will discuss the operation of unipolar-induction, spin-orbit synchronisation and life-span of unipolar induction UCDs in more detail.

\section{ORBITAL EVOLUTION OF UNIPOLAR-INDUCTOR COMPACT BINARIES}

\subsection{Spin-orbit coupling}

In the unipolar inductor model, the system is asynchronous, and the orbital evolution is described neither by Equation (9) nor (10) in Section 2. Additional energy dissipation needs to be taken into account. Without loss of generality, we consider the non-magnetic white dwarf as tidally locked to synchronous rotation with the orbit. This is justified if the secondary white dwarf is close to filling its Roche lobe. Through spinorbit coupling, energy and angular momentum are transferred between the binary orbit and the spin of the magnetic white dwarf, but the transfer rates depend on the orbital properties and the dissipation processes. 
We may define a quantity

$$
W^{*} \equiv \frac{W}{(1-\alpha)^{2}}
$$

This quantity is independent of the asynchronism parameter $\alpha$, and it gives the timescale on which the system achieves spin-orbit synchronism. The two essential equations governing the spin-orbit evolution are

$$
\begin{aligned}
& \frac{\dot{\omega}_{\mathrm{o}}}{\omega_{\mathrm{o}}}=\frac{\dot{E}_{\mathrm{gw}}}{g\left(\omega_{\mathrm{o}}\right)}\left[1-(1-\alpha) \frac{W^{*}}{\dot{E}_{\mathrm{gw}}}\right], \\
& \frac{\dot{\alpha}}{\alpha}=-\frac{\dot{E}_{\mathrm{gw}}}{g\left(\omega_{\mathrm{o}}\right)}\left[1-(1-\alpha)\left(1+\frac{g\left(\omega_{\mathrm{o}}\right)}{\alpha I_{1} \omega_{\mathrm{o}}^{2}}\right) \frac{W^{*}}{\dot{E}_{\mathrm{gw}}}\right]
\end{aligned}
$$

(Wu et al. 2002), where

$$
\begin{aligned}
g\left(\omega_{\mathrm{o}}\right) & =-\frac{1}{3}\left[\frac{q^{3}}{1+q} G^{2} M_{1}^{5} \omega_{\mathrm{o}}^{2}\right]^{1 / 3}\left[1-\frac{6}{5}(1+q) f\left(\omega_{\mathrm{o}}\right)\right] \\
& =-\frac{1}{3} G^{2 / 3} M_{\mathrm{chirp}}^{5 / 3} \omega_{\mathrm{o}}^{2 / 3}\left[1-\frac{6}{5}(1+q) f\left(\omega_{\mathrm{o}}\right)\right] .
\end{aligned}
$$

The structure factor $f\left(\omega_{0}\right)$ is

$$
f\left(\omega_{\mathrm{o}}\right)=\left[\frac{R_{2}^{3} \omega_{\mathrm{o}}^{2}}{G\left(M_{1}+M_{2}\right)}\right]^{2 / 3} .
$$

The moment of inertia of the magnetic white dwarf, $I_{1}=2 \eta M_{1} R_{1}^{2} / 5$, and the parameter $\eta$ depends on the density distribution and shape of the white dwarf. For spherical stars with a uniform density, $\eta=1$. Note that by setting $W^{*}=0$ and considering $\lim f\left(\omega_{\mathrm{o}}\right) \rightarrow 0$, we can recover the expression of $\omega_{\mathrm{o}} / \omega$ for the case with no spin-orbit coupling (Eq.96).

\subsection{Life span of unipolar-inductor compact binaries}

The right hand side of Equation (21) is dominated by the final term in the bracket. The synchronisation of the system due to electrical dissipation in the unipolar-inductor circuit is essentially governed by the equation

$$
\alpha \approx 1-\left(1-\alpha_{0}\right) \exp \left[-\frac{t}{\tau_{\mathrm{ui}}}\right]
$$

where

$$
\tau_{\mathrm{ui}}=\frac{I_{1} \omega_{\mathrm{o}}^{2}}{W^{*}}
$$

is the synchronisation (unipolar-induction) timescale. An approximate expression for $\alpha_{0}$ as a function of $M_{1}$ and $M_{2}$ can be obtained by solving Equation (20) for $\alpha$, with

$$
\alpha_{0} \sim 1-\frac{1}{W^{*}}\left[\dot{E}_{\mathrm{gw}}-g\left(\omega_{\mathrm{o}}\right) \frac{\dot{\omega}_{\mathrm{o}}}{\omega_{\mathrm{o}}}\right]
$$

(Willes \& Wu, unpublished). The lifetime of the system is limited by gravitational radiation loss, with the merging timescale for the binary system given by

$$
\tau_{\mathrm{gw}}=\frac{a_{0}^{4}}{4 \Theta}
$$


where $a_{0}$ is the initial binary orbital separation, and

$$
\begin{aligned}
\Theta & =a^{3} \dot{a} \\
& =\frac{64}{5} \frac{G^{3}}{c^{5}}\left[M_{1} M_{2}\left(M_{1}+M_{2}\right)\right]
\end{aligned}
$$

(Peters 1964; see also Landau \& Liftshitz 2002).

The synchronisation timescale appears to be very short, with $\tau_{\mathrm{ui}}<1000 \mathrm{yr}$, for a range of combinations of white-dwarf mass $\left(M_{1}, M_{2}\right)$ and magnetic moment $(\mu)$. One might be concerned that unipolar-induction systems can be X-ray sources over only a small fraction of the binary system lifetime $\tau_{\mathrm{gw}}$, which is the timescale for white-dwarf coalescence due to gravitational radiation losses. The apparently short X-ray emission phase could pose problems for the detectability of these systems, as pointed out by Barros et al. (2005). A possible resolution is to invoke a mechanism, such as intermittent mass transfer, which causes repeated episodes of spin-orbit de-synchronisation over the system lifetime. While this is possible, it is not always necessary. In the parameter regimes of UCDs, the unipolar induction phase can operate and produce $\mathrm{X}$-ray pulses over the system lifetime before coalescence occurs, even when $\tau_{\mathrm{ui}} \ll \tau_{\mathrm{gw}}$.

This phenomenon can be illustrated with the following example. Consider a system with white-dwarf masses $M_{1}=0.7 \mathrm{M}_{\odot}$ and $M_{2}=0.345 \mathrm{M}_{\odot}$ and with the primary white dwarf having a magnetic moment $\mu=10^{30} \mathrm{G} \mathrm{cm}^{3}$. For these parameters, the initial value of the asynchronism parameter required to fit the observed orbital period $P_{\mathrm{o}}$ and period derivative $\dot{P}_{\mathrm{o}}$ for RX J0806+15 is $\alpha_{0}=0.95$. Figure 6 shows the evolution of $\alpha$ over a 50000 year period. The synchronisation timescale of the system $\tau_{\text {ui }} \sim 5000 \mathrm{yr}$. After a brief unipolar-inductor phase $\left(t>\tau_{\mathrm{ui}}\right)$, the driver of system evolution is taken over by the gravitational radiation loss. However, the system has not achieved synchronism by the end of the unipolar-inductor phase $(\alpha \neq 1)$. During the subsequent slow evolution, gravitational radiation loss ensures that the system remains asynchronous over the remaining time span until the eventual coalescence of the two white dwarfs. Note that the rate of change of $\alpha$ is effectively zero (in comparison to the fast evolution of $\alpha$ during the unipolarinductor phase), despite the fact that the system is in an asynchronous state. The value of $\alpha$ at the "end" of the unipolar-inductor phase can be estimated by equating the first and last terms on the right-hand side of Equation 21 (i.e. by setting $\dot{\alpha}=0$, and where $g\left(\omega_{\mathrm{o}}\right) /\left(\alpha I_{1} \omega_{\mathrm{o}}^{2}\right) \gg 1$ ), yielding

$$
\alpha_{\mathrm{gw}}=\frac{\chi}{1+\chi}
$$

where

$$
\chi=\frac{g\left(\omega_{\mathrm{o}}\right)}{\dot{E}_{\mathrm{gw}} \tau_{\mathrm{ui}}} .
$$

For these parameters, $\alpha_{\mathrm{gw}}=0.97$, which is in approximate agreement with the value of $\alpha$ at the end of the unipolar-inductor phase, $t \sim 5000 \mathrm{yr}$ (top panel, Fig. 6). The system remains unsynchronised, with $\alpha \approx 0.98$, over a period of $t \sim 50000 \mathrm{yr}$. Throughout the evolution, the footpoint luminosity (electrical dissipation) $W$ exceeds $10^{33} \mathrm{erg} \mathrm{s}^{-1}$ (bottom panel, Fig. 6).

We note that for some parameters, a system can achieve a high degree of synchronisation on a short timescale. For instance if we consider different masses for the white dwarf, say $M_{1}=0.7 \mathrm{M}_{\odot}$ and $M_{2}=$ $0.1 \mathrm{M}_{\odot}$, then the system is almost completely synchronised and $\alpha_{\mathrm{gw}}$ reaches 0.998 within $1500 \mathrm{yr}$, and the corresponding footpoint luminosity $W$ falls below $10^{32} \mathrm{erg} \mathrm{s}^{-1}$ soon after this time. Here we have demonstrated that over a certain range of parameters, the unipolar-inductor model can sustain intense X-ray emissions over the entire lifetime of the system, rather than the much shorter unipolar-inductor timescale.

Compact white-dwarf pairs are strong sources of gravitational radiation. They are populous in the solar neighbourhood and are among the first to be detected by the gravitational wave observatory LISA (see Cutler, Hiscock \& Larson 2003; Nelemans 2003; Nelemans, Yugelson \& Portegies Zwart 2004; Kopparapu $\&$ Tohline 2007). These sources can be calibrators of the experiments or pests that cause foreground contamination of the weaker cosmological signals. In order to have these sources detected and subtracted, one needs good waveform templates of the gravitational radiation that they emit. As illustrated above, the unipolar-induction can persist throughout the lifetime of a UCD until the two white dwarfs coalesce. The 

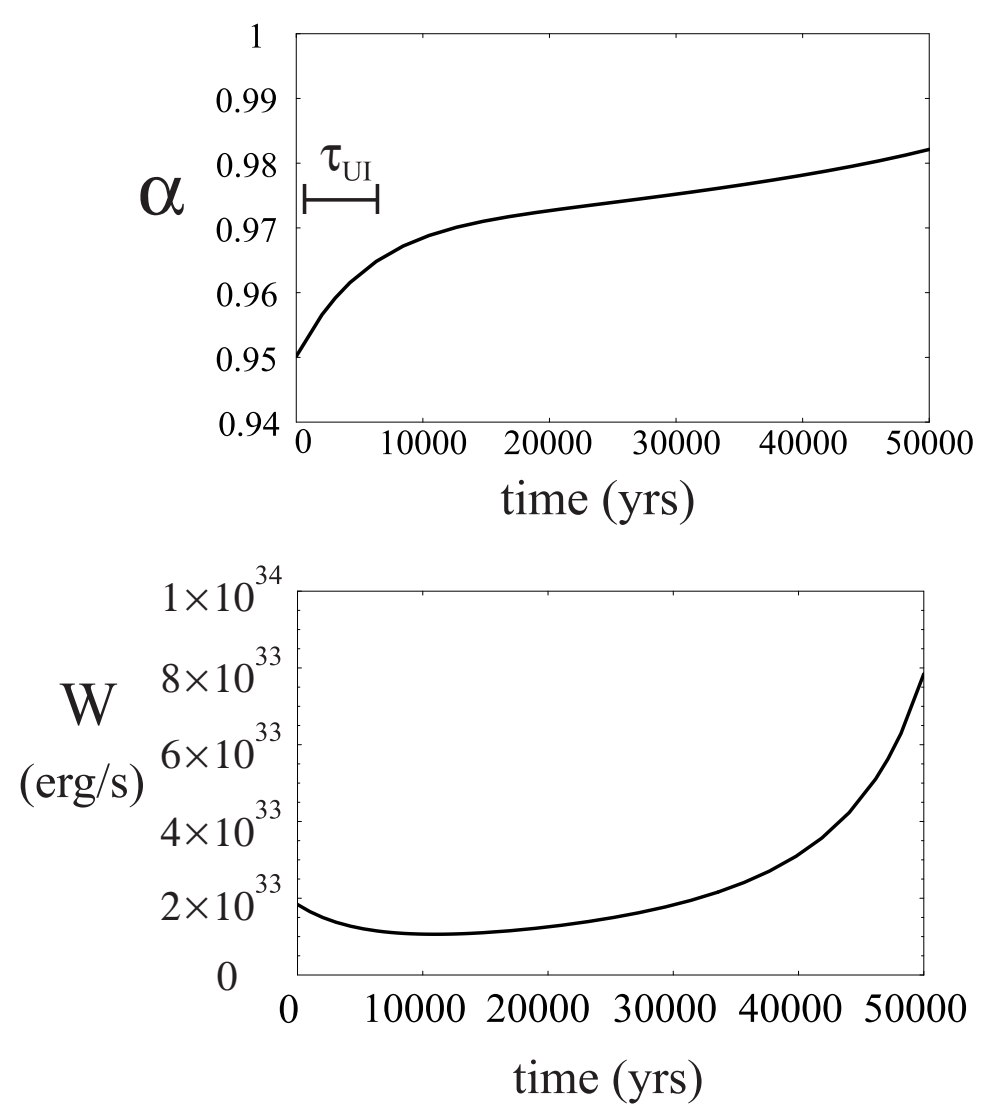

Fig. 6 (Top) Evolution of the asynchronicity parameter $\alpha$ for a compact white-dwarf pair with $M_{1}=0.7 \mathrm{M}_{\odot}$ and $M_{2}=0.345 \mathrm{M}_{\odot}$ and $\mu=10^{30} \mathrm{G} \mathrm{cm}^{3}$. The orbital period and its time derivative take the values derived for RX J0806+15 (see Israel et al. 2003; Hakala et al. 2003). The evolution is characterized by a rapid phase (up to $t \sim \tau_{\mathrm{ui}}$ ) followed by a slow phase where the evolution is controlled the dynamics of the orbital decay associated with gravitational radiation losses. For this set of parameters, the system would not be completely synchronised when entering the slow phase. (Bottom) Evolution of the footpoint luminosity (electrical dissipation) $W$ associated with $\alpha$ from the top panel. The value of $W$ remains above the level of $\sim 10^{33} \mathrm{erg} \mathrm{s}^{-1}$ throughout the entire binary-system lifetime, and at the later evolutionary stage it even increases despite the spin and orbit becoming more synchronised. (Diagrams provided by A. Willes.)

orbital evolution is determined by the energy loss due to gravitational radiation and electrical dissipation. Without taking into account the contribution of unipolar induction, the gravitational wave signals of a UCD can become de-coherent on timescales as short as days, thus posing serious problems in the UCD detection.

\section{ELECTRON-CYCLOTRON MASER EMISSION}

UCDs are potential electron-cyclotron maser sources. The two distinguishable characteristics of electroncyclotron masers are high brightness temperature and almost $100 \%$ circular polarization. The operation of electron-cyclotron masers requires a population inversion in the electron distribution and a magnetised plasma in which the electron-cyclotron frequency $\Omega_{\mathrm{e}}$ exceeds the plasma frequency $\omega_{\mathrm{p}}$ (e.g. Dulk 


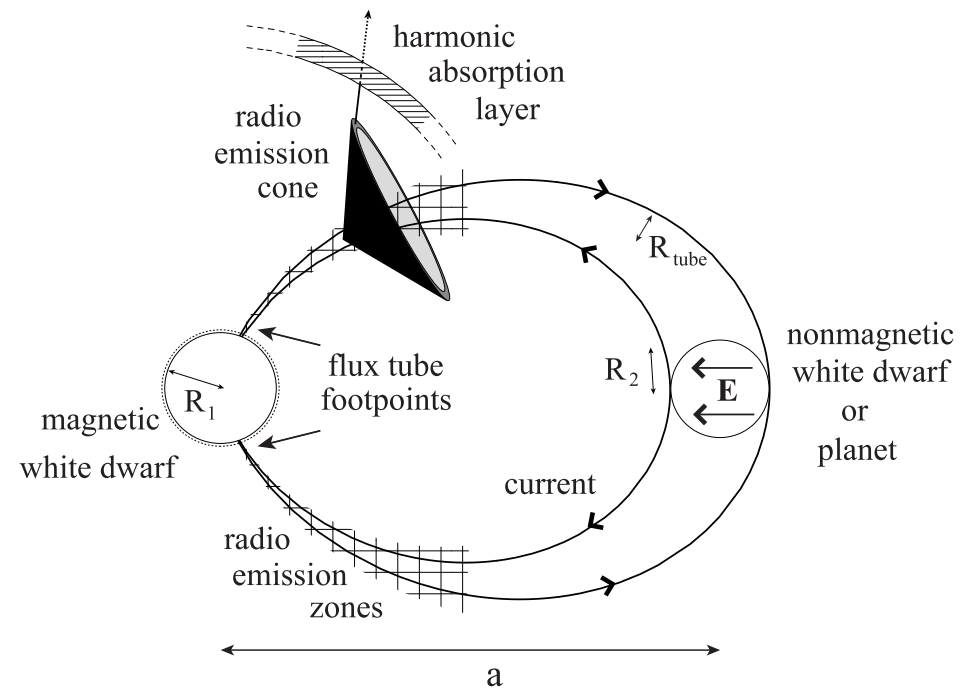

Fig. 7 An illustration to show the regions where electron-cyclotron masers would emit in a compact white-dwarf pair or a white-dwarf planetary system.

1985). These two conditions can be satisfied in a variety of astronomical settings. The first condition can be achieved in the presence of a loss-cone or a shell electron distribution. These distributions are kinetically unstable, and the instability provides the free energy for the generation of electron-cyclotron masers (Wu \& Lee 1979; Melrose \& Dulk 1982; Pritchett 1984; Melrose 2005; Treumann 2006) A loss-cone electron distribution arises when an electron pitch-angle anisotropy develops within a magnetic flux tube with converging field lines at each foot point. Large pitch angle electrons are magnetically reflected, whereas small-pitch-angle electrons are lost through collisions with high density plasma at the foot of the magnetic flux tube. The second condition is satisfied in magnetized plasmas with a relatively low electron density and/or a high magnetic field strength.

In Jupiter and Io, electron cyclotron masers are emitted from the current-carrying electrons in the Io magnetic flux tube. The observed high brightness temperatures ( $\gtrsim 10^{17} \mathrm{~K}$, Dulk 1970), $100 \%$ circularly polarization (Dulk, Lecacheux \& Leblanc 1992) and the radiation beaming pattern in the radio emission from Jupiter-Io are characteristics of electron-cyclotron masers. The anti-correlation between infrared footpoint emission and Io-controlled Jovian decametric radiation indicates that the masers are driven by reflected electrons (Connerney et al. 1993). The presence of reflected electrons in a loss-cone distribution is also consistent with the observation of negative frequency drifts in the fine-frequency structure of Jovian decametric radiation S-bursts (Ellis 1974).

The operation of unipolar induction and the similarity of configurations between a unipolar inductor UCD and the Jupiter-Io system imply that loss-cone instability may develop in the magnetic flux tubes in a UCD (see Fig. 7). The main differences between a UCD and the Jupiter-Io system are probably the energetics of the streaming electrons in the current circuits, which participate in developing the loss-cone instability, and the amount of thermal electrons present in the system, which could suppress the maser process. A model of electron-cyclotron masers from white-dwarf pairs can be constructed in the unipolarinductor framework (see Willes \& Wu (2004) and Willes, Wu \& Kuncic (2004) for details). The predicted flux densities of electron-cyclotron masers from UCDs with parameters are shown in Figure 8 . For parameters similar to those derived for RX J1914+24 and RX J0806+15, the electron-cyclotron masers are 


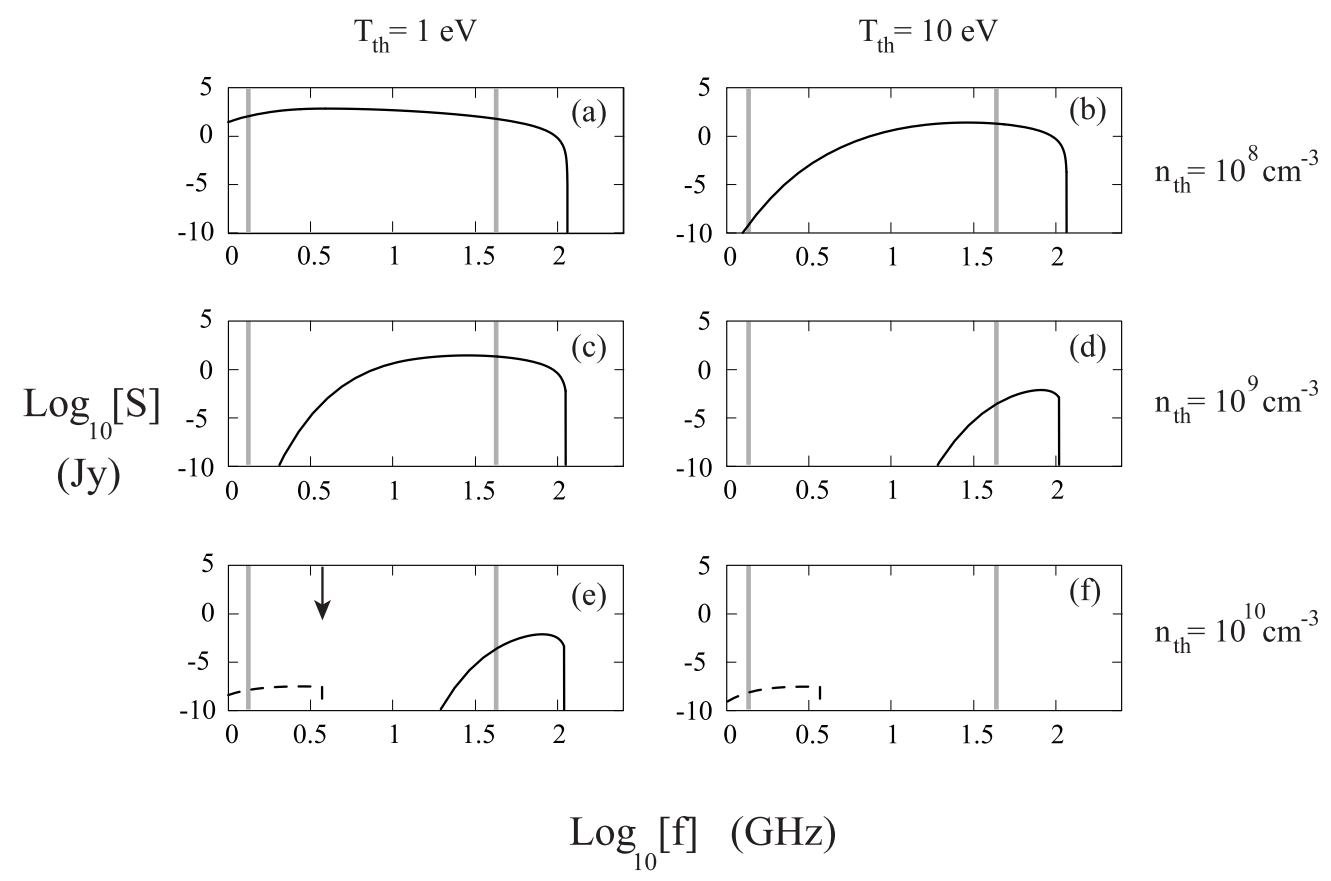

Fig. 8 Peak flux densities of electron-cyclotron maser (in the radio wave-bands) from unipolarinductor UCDs (maximised over emission angle). The system parameters of the UCD are whitedwarf masses $M_{1}=0.7 \mathrm{M}_{\odot}$ and $M_{2}=0.5 \mathrm{M}_{\odot}$, orbital period $P_{\mathrm{o}}=540 \mathrm{~s}$, magnetic moment of the primary white dwarf $\mu=10^{31} \mathrm{G} \mathrm{cm}^{3}$ and degree of asynchronism of 1 part in 1000 . The losscone parameters are the temperature $k T=1 \mathrm{keV}$, the electron number density $n_{\mathrm{lc}}=10^{9} \mathrm{~cm}^{-3}$, and the edge width $\Delta \alpha=0.05$ (see Willes \& Wu 2004). Left and right columns correspond to cases with thermal electrons of temperatures $k T_{\mathrm{th}}=1$ and $10 \mathrm{eV}$ respectively. Panels from top to bottom correspond to thermal electron number density $n_{\mathrm{th}}=10^{8}, 10^{9}$ and $10^{10} \mathrm{~cm}^{-3}$ respectively. The x-mode emission is represented by solid lines, and the o-mode emission by dashed lines. The vertical grey lines mark the VLA observing frequencies of 1.465 and $43 \mathrm{GHz}$.

observable using current instruments such as the radio telescopes ATCA and VLA. A radio survey would identify unipolar-inductor UCDs which emit only weak X-rays or have a very soft X-ray spectrum.

Note that a recent search for electron-cyclotron masers from UCDs (Ramsay et al. 2007) revealed a 5- $\sigma$ source at the position of RX J0806+15. The inferred brightness temperature exceeded $10^{18} \mathrm{~K}$ and the upper limit for circular polarization was about $50 \%$.

\section{BEYOND ULTRA-COMPACT DOUBLE DEGENERATES}

\subsection{White-dwarf planetary systems}

Electron-cyclotron masers can also be generated in astronomical binaries having similar configurations for electric currents and magnetic fields as a unipolar-inductor UCD. An example is a magnetic white dwarf with an orbiting terrestrial planet with a metallic core (Li, Ferrario \& Wickramasinghe 1998). The metallic core is a good electric conductor. It provides the e.m.f. to drive the current flow. A unipolar-inductor whitedwarf planetary system differs from a unipolar-inductor UCD by replacing the non-magnetic white dwarf with a less massive terrestrial planet. Electron-cyclotron maser generation is determined by the magnetic field and electric current configurations, and the charge acceleration processes. The strength of the masers 

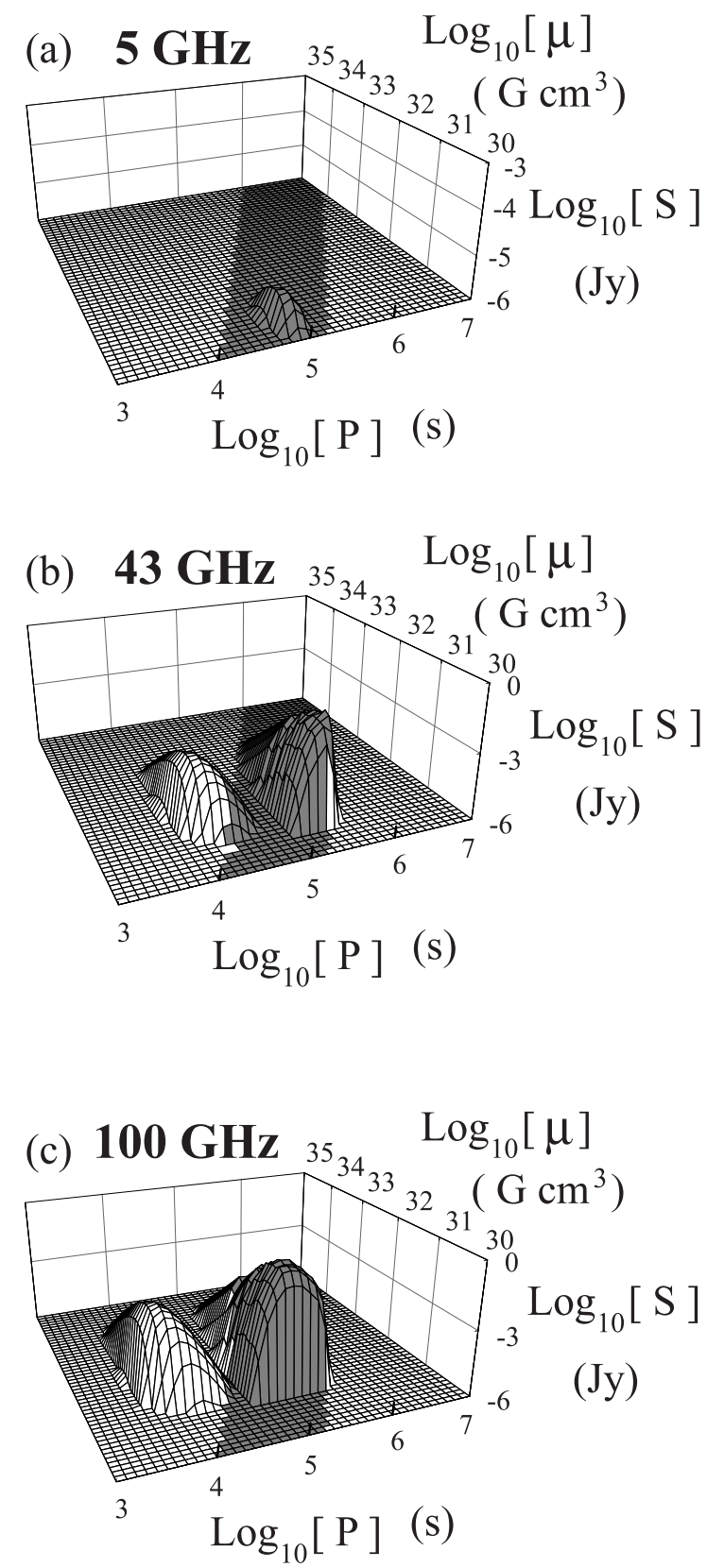

Fig. 9 The flux density of loss-cone electron-cyclotron masers at 5, 43 and $100 \mathrm{GHz}$ from a UI white-dwarf planetary system. The magnetic moment of the magnetic white dwarf $\mu=$ $10^{30} \mathrm{G} \mathrm{cm}^{3}$, the number density of the loss-cone electron population is $10^{7} \mathrm{~cm}^{-3}$, and the mean energy of the electrons is $1 \mathrm{keV}$. The distance to the source is $100 \mathrm{pc}$. The gray band denotes the region in the parameter space where detection is expected for the current instrumentation. 
has a very strong dependence on the system size but a weak dependence on the system mass. Because a terrestrial planet and a white dwarf have similar sizes, electron-cyclotron masers in white-dwarf planetary systems can be as strong as in UCD (Willes \& Wu 2004, 2005). The predicted flux densities of loss-cone electron-cyclotron masers from a unipolar-inductor white-dwarf planetary system can exceed $0.1 \mathrm{Jy}$ for certain sensible system parameters (see Fig. 90).

Can white-dwarf planetary systems be formed? White dwarfs are remnants of solar-like and low-mass stars. After evolving beyond the main-sequence and the giant phases, the sun will become a white dwarf. For a solar-like system, if the inner planets can survive being engulfed by the inflated stellar envelope during the red-giant/asymptotic-giant phases, the system will become a white-dwarf planetary system (see discussions in Willes \& Wu 2005). Provided that the terrestrial planets spiral in sufficiently close to the white dwarf so that efficient unipolar induction can operate, a loss-cone or shell electron distribution may develop, leading to the emission of electron-cyclotron masers. A population synthesis (Willes \& Wu 2005) suggested that there would be about five systems expected to be detected by VLA at $43 \mathrm{GHz}$, about 20 systems by SKA at $20 \mathrm{GHz}$, and about 100 systems by ALMA at $100 \mathrm{GHz}$.

\subsection{Einstein-Laub effect in compact binaries}

Einstein and Laub (1908) pointed out that a magnetic dipole moment moving in a constant velocity would develop an electric dipole moment, i.e.

$$
\boldsymbol{d}=\boldsymbol{\beta} \times \boldsymbol{\mu},
$$

where $\boldsymbol{E}$ is the electric field, $\boldsymbol{B}$ the magnetic induction, $\boldsymbol{\beta}$ the velocity normaised to the speed of light in vacuum, $\boldsymbol{d}$ the electric dipole moment, and $\boldsymbol{\mu}$ the magnetic dipole moment. This essentially says that electrodynamics is a restrictive case of special relativity. However, it is not easy to set up an experiment in which a strong magnet moves at a relativistic speed so as to induce a measurable electric dipole moment.

In a slab of insulating material moving with a constant velocity, we would expect an electric polarisation $P$, given by

$$
\boldsymbol{P}=\frac{\epsilon-1}{4 \pi}(\boldsymbol{E}+\boldsymbol{\beta} \times \boldsymbol{B})+(\boldsymbol{\beta} \times \boldsymbol{m}),
$$

where $\boldsymbol{m}$ is the magnetic polarisation and $\epsilon$ is the dielectric constant. Wilson and Wilson (1913) conducted a rotating-cylinder experiment, which appeared to have verified this effect. However, the interpretation of their results and whether the experiment is a validation of the effect has been under debate (see Pellegrini \& Swift 1995; Weber 1997; Krotkov et al. 1999; Hertzberg et al. 2001). The arguments centre on the fact that a spinning device was used in the Wilson \& Wilson experiment while rotation is not equivalent to translational motion.

The Einstein-Laub effect was subsequently verified in a molecular beam experiment (Sangster et al. $1993 ; 1995)$, which was designed for other scientific objectives. In the experiment, a beam of magnetically polarised thallium fluoride molecules (the magnetic dipoles with moments $\boldsymbol{\mu}$ ) with a velocity $\boldsymbol{\beta}$ was sent through a region of constant electric field $\boldsymbol{E}$. By measuring the Ahronov-Casher phase shift, which is given by

$$
\begin{aligned}
\hbar \phi_{a b} & =\int_{a}^{b} d t(\boldsymbol{\mu} \times \boldsymbol{E}) \cdot \boldsymbol{\beta} \\
& =\int_{a}^{b} d t(\boldsymbol{\beta} \times \boldsymbol{\mu}) \cdot \boldsymbol{E} \\
& =\int_{a}^{b} d t \boldsymbol{d} \cdot \boldsymbol{E},
\end{aligned}
$$

where $\hbar$ is the reduced Planck constant. Sangster et al. (1995) deduced the interaction energy and used it to infer the induced dipole moment $\boldsymbol{d}$. The result agreed with the theoretical prediction by relativity to within $2 \%$. 
We note that the Einstein-Laub effect in a rotating device is manifested in compact binaries. Consider a magnetised white dwarf rotating in a tight orbit around another compact object. The white dwarf has a magnetic moment $\boldsymbol{\mu}$. For simplicity, the magnetic moment $\boldsymbol{\mu}$ is perpendicular to the orbital angular velocity $\boldsymbol{\omega}_{\mathrm{o}}$. Moreover, the white-dwarf spin is magnetically locked into synchronous rotation with the orbit, as in the AM Herculis binaries. The orbital rotation of the white dwarf would then induce a spinning electric dipole moment with a magnitude

$$
d=\frac{\mu r_{\mathrm{o}} \omega_{\mathrm{o}}}{c},
$$

where $r_{\mathrm{o}}$ is the radius of the white-dwarf orbit with respect to the centre of mass of the binary. A spinning electric dipole is known to emit electromagnetic waves, The radiative power is given by

$$
L=\frac{2}{3} \frac{\ddot{d}^{2}}{c^{3}}=\frac{2}{3} \frac{\mu^{2} r_{\mathrm{o}}^{2} \omega_{\mathrm{O}}^{6}}{c^{5}} .
$$

For a binary with $P_{\mathrm{o}} \sim 300 \mathrm{~s}, r_{\mathrm{o}} \sim 10^{10} \mathrm{~cm}$, and a white dwarf with $\mu \sim 10^{33} \mathrm{G} \mathrm{cm}^{3}$, the radiative power will be $L \sim 2.3 \times 10^{23} \mathrm{erg} \mathrm{s}^{-1}$. This value is similar to that of thermal emission from a spherical body with a temperature of about $300 \mathrm{~K}$ and an Earth-sized radius. However, $r_{\mathrm{o}} \propto a_{\mathrm{o}} \propto \omega_{\mathrm{o}}^{-2 / 3}$, implying that $L \propto \omega_{\mathrm{o}}^{14 / 3}$ (cf. $L_{\mathrm{gw}}=\dot{E}_{\mathrm{gw}} \propto \omega_{\mathrm{o}}^{10 / 3}$ for gravitational radiation). For a system with $P_{\mathrm{o}} \sim 5 \mathrm{~s}$ (possible for two neutron stars in a merging process), the expected radiative power would exceed $10^{31} \mathrm{erg} \mathrm{s}^{-1}$, which would have some observational consequences.

\section{SUMMARY}

Ultra-compact double degenerates contain two compact stars revolving around each other in a very tight orbit. The proximity of the two stars allows efficient magnetic coupling between the stellar spins and the orbital rotation. The presence of unipolar induction in compact binaries could greatly affect the orbital dynamics in compact binaries, leading to observational consequences in gravitational radiation as well as in electromagnetic radiation domains. Unipolar-inductor compact binaries are possible strong sources of electron-cyclotron masers. The maser model for unipolar-inductor ultra-compact double degenerate can be applied to white-dwarf planetary systems. Einstein-Laub effects may be observable in compact binaries with extremely short orbital periods.

Acknowledgements I thank Jingxiu Wang for the suggestion to write this review and continuous encouragement throughout the writing process. Andrew Willes, Mark Cropper and Gavin Ramsay have been my collaborators in various research projects on compact binary systems. They have contributed much to the science discussed in this article. I thank Andrew Willes for providing results of his unpublished work on the evolutionary history of unipolar-inductor UCDs and, in particular, Figure 6, I also thank Gian-Luca Israel for providing Figure 5, and Ziri Younsi and Curtis Saxton for reading through the manuscript.

\section{References}

Alfvén H., Fälthammar C.-G., 1963, Cosmic Electrodynamics (London: Oxford University Press)

Assis A. K. T., 2000, Phys. Rev., E62, 7544

Barros S. C. C., et al., 2005, MNRAS, 357, 1306

Barros S. C. C., et al., 2007, MNRAS, 374, 1334

Belczynski K., Taam R. E., 2004, ApJ, 603, 690

Belczynski K., Kalogera V., Rasio F. A., Taam R. E., Zezas A., Bulik T., Maccarone T. J., Ivanova N., 2008, ApJS, 174, 223

Blandford R. D., 2000, Physica Scripta, T85, 91

Campbell C. G., 1983, MNRAS, 205, 1031

Campbell C. G., 1999, MNRAS, 306, 307 
Chanmugam G., 1992, ARA\&A, 30, 143

Chanmugam G., Brecher K., 1985, Nature, 313, 764

Chanmugam G., Dulk G. A., 1982, ApJ, 255, L107

Chanmugam G., Wagner R. L., 1977, ApJ, 213, L13

Clarke J. T., et al., 1996, Science, 274, 404

Connerney J. E. P., Baron R., Satoh T., Owen T., 1993, Science, 262, 1035

Cropper M., Harrop-Allin M. K., Mason K. O., Mittaz J. P. D., Potter S. B., Ramsay G., 1998, MNRAS, 293, L57

Cropper M., Ramsay G., Wu K., Hakala P., 2004, ASPC, 315, 324

Cropper M. et al., 1989, MNRAS, 236, 29

Cutler C., Hiscock W. A., Larson S. L., 2003, Phys. Rev. D67, 024015

Dall'Osso S., Israel G. L., Stella L., 2006, A\&A, 447, 785

Dall'Osso S., Israel G. L., Stella L., 2007, A\&A, 464, 417

Davis P., Kolb U., Willems B. T., Gänsicke B. T., 2008, MNRAS, 389, 1563

Dulk G. A., 1970, ApJ, 159, 671

Dulk G. A., 1985, ARA\&A, 23, 169

Dulk G. A., Lecacheux A., Leblanc Y., 1992, A\&A, 253, 292

Eggleton P. P., 1983, ApJ, 268, 368

Einstein A., Laub J., 1908, Ann. Phys. (Leipzig), 26, 532

Ellis G. R. A., 1974, PASA, 2, 236

Ferreira J. M., Mendoza-Briceño C. A., 2005, A\&A, 433, 1055

Feynman R., Leighton R. B., Sands M., 1964, The Feynman Lectures on Physics Vol II (Reading: Addison-Wesley)

Goldreich P., Julian W. H., 1969, ApJ, 157, 869

Goldreich P., Lynden-Bell D., 1969, ApJ, 156, 59

Guala-Valverde J., Mazzoni P., Achilles R., 2002, Am. J. Phys., 70, 1052

Hakala P., et al., 2003, MNRAS, 343, L10

Han Z., 1998, MNRAS, 296, 1019

Hertzberg J. B., Bickman S. R., Hummon M. T., Krause D., Peck S. K., Hunter L. R., 2001, Am. J. Phys., 69, 648

Israel G. L., Panzera M. R., Campana S., Lazzati D., Covino S., Tagliaferri G., 1999, A\&A, 349, L1

Israel G. L., et al., 2002, A\&A, 386, L13

Israel G. L., et al., 2003, ApJ, 598, 492

Kelley A. G., 1999, Phys. Essays, 12, 372

Komissarov S. S., 2004, MNRAS, 350, 427

Kopparapu R. K., Tohline J. E., 2007, ApJ, 655, 1025

Krotkov R. V., Pellegrini G. N., Ford N. C., Swift A. R., 1999, Am. J. Phys., 67, 498

Laine R. O., Lin D. N. C., Dong S., 2008, ApJ, 685, 521

Landau L. D., Lifshitz E. M., 2002, The Classical Theory of Fields (4th ed., Oxford: Butterworth-Heinemann)

Lewin W. H. G., van Paradijs J., van den Heuvel E. P. J., 1997, X-ray Binaries, (Cambridge: Cambridge University Press)

Li J., Ferrario L., Wickramasinghe D. T., 1998, ApJ, 503, L151

Li J. K., Wu K., Wickramasinghe D. T., 1994a, MNRAS, 268, 61

Li J. K., Wu K., Wickramasinghe D. T., 1994b, MNRAS, 270, 769

Marsh T. R., Steeghs D., 2002, MNRAS, 331, L7

Mason P. A., Ramsay G., Andronov I., Kolesnikov S., Shakhovskoy N., Pavlenko E., 1998, MNRAS, 295, 511

Melrose D. B., 2005, PASA, 22, 144

Melrose D. B., Dulk, G. A., 1982, ApJ, 259, 844

Miller A., 1981, Ann. Sci. NY, 38, 155 
Montgomery H., 1999, Eur. J. Phy., 20, 271

Motch C., Haberl F., Guillout P., Pakell M., Reiasch K., Krätter J., 1996, A\&A, 307, 459

Nelemans G., 2005, ASPC, 330, 27

Nelemans G. 2003, Class. Quantum Grav., 20, S81

Nelemans G., Yungelson L. R., Portegies Zwart S. F., 2004, MNRAS, 349, 181

Nelemans G., Yungelson L. R., Portegies Zwart S. F., Verbunt F., 2001, A\&A, 365, 491

Norton A. J., Haswell C. A., Wynn G. A., 2004, A\&A, 419, 1025

Paczynski B., 1971, ARA\&A, 9, 183

Piddington J. H., Drake J. F., 1968, Nature, 217, 935

Pellegrini G. N., Swift A. R., 1995, Am. J. Phys., 63, 694

Peters P. C. 1964, Phys. Rev., 136, B1224

Postnov K. A., Yungelson L. R., 2006, Living Rev. Relativity, 9, 6

Pritchett P. L., 1984, J. Geophys. Res., 89, 8957

Punsly B., 2001, Black Hole Gravitohydromagnetic (Berlin: Springer-Verlag)

Ramsay G., Brocksopp C., Wu K., Slee B., Saxton C. J., 2007, MNRAS, 382, 461

Ramsay G., Cropper M., Wu K., Mason K. O., Hakala P., 2000, MNRAS, 311, 75

Ramsay G., et al., 2002, MNRAS, 333, 575

Ramsay G., et al., 2005, MNRAS, 357, 49

Retter A., Richards M. T., Wu K., 2005, ApJ, 621, 417

Richards M. T., Albright G. E., 1993, ApJS, 88, 199

Roelofs G. H. A., Nelemans G., Groot P. J., 2007, MNRAS, 382, 685

Sangster K., Einds E. A., Barnett S. M., Riis E., 1993, Phys. Rev. Lett., 71, 364

Sangster K., Einds E. A., Barnett S. M., Riis E., Sinclair A. G., 1995, Phys. Rev. A, 51, 1776

Schmidt G. D., Smith P. S., 1995, ApJ, 448, 305

Shatskii A. A., 2003, Astronomy Letters, 29, 1153

Shatskii A. A., Kardashev N. S., 2002, Astronomy Reports, 46, 639

Solheim J.-E., 1995, Baltic Astronomy, 4, 363

Spitzer L., Härm R., 1953, Phys. Rev., 89, 977

Steeghs D. et al., 2006, ApJ, 649, 382

Strohmayer T. E., 2002, ApJ, 581, 577

Strohmayer T. E., 2003, ApJ, 593, L39

Strohmayer T. E., 2004, ApJ, 610, 416

Strohmayer T. E., 2005, ApJ, 627, 920

Strohmayer T. E., 2008, ApJ, 670, L109

Treumann R. A., 2006, Astron. Astrophys. Rev., 13, 229

Uchida Y., Sakurai T., 1983, Activity in Red Dwarf Stars, ed. M. Rodono, P. Byrne, IAUC, 71, p.629

Visvanathan N., Wickramasinghe D. T., 1981, MNRAS, 196, 275

Warner B., 1995, Cataclysmic Variable Stars (Cambridge: Cambridge University Press)

Weber T. A., 1997, Am. J. Phys., 65, 946

Wickramasinghe D. T., Ferrario L., 2000, PASP, 112, 873

Wickramasinghe D. T., Wu K., 1991, MNRAS, 253, 11P

Willes A. J., Wu K., 2004, MNRAS, 348, 285

Willes A. J., Wu K., 2005, A\&A, 432, 1091

Willes A. J., Wu K., Kuncic Z., 2004, PASA, 21, 248

Williams G., 1983, ApJS, 53, 523

Wilson M., Wilson H. A., 1913, Proc. Roy. Soc. London, A89, 99 
Wood M. A., 2009, MNRAS, 395378

Wu C. S., Lee L. E., 1979, ApJ, 231, 621

Wu K., 1997, ASPC, 121, 283

Wu K., 2000, Space Science Review, 93, 611

Wu K., Wickramasinghe D. T., 1993, MNRAS, 260, 141

Wu K., Ramsay G., Willes A., 2008, ChJAS, 8, 169

Wu K., Cropper M., Ramsay G., Sekiguchi K., 2002, MNRAS, 331, 221

Zarka Ph., 2007, Planetary and Space Science, 55, 598

This paper was prepared with the RAA LATEX macro v1.2. 